\title{
Measurement of Force Curve due to Electrostatic Charge on a Single Particle using Atomic Force Microscope
}

\author{
Tatsushi MATSUYAMA*, Masa-aki OHTSUKA and Hideo YAMAMOTO \\ Department of Environmental Engineering for Symbiosis \\ Faculty of Engineering \\ Soka University ${ }^{1}$
}

\begin{abstract}
A single particle is brought into contact with a metal target, and the force acting on the particle is measured by using atomic force microscope (AFM). By focusing on measuring the force 'curve', rather than looking only at the maximum adhesive force, electrostatic interaction was successfully observed by separating other interactions such as liquid bridge and intermolecular forces. To evaluate the force curve, the force curve with an approximation of disk-to-disk interaction based on image force was numerically calculated and a good analytical approximation was developed. The fact of successful agreement between the observed force curve and the theory revealed that the force curve observed can be surely attributed to the electrostatic interaction, and that the amount of charge on the particle and the radius of the charged (contact) area can be estimated from the analysis. The order of magnitude of the measured charge density was $10^{-2} \mathrm{C} / \mathrm{m}^{2}$, which is much greater than that obtained with impact charging experiment as $10^{-4} \mathrm{C} / \mathrm{m}^{2}$. From this it was concluded that the force curve measurement with AFM can capture the net amount of the charge generated before charge relaxation due to gas discharge taking place. In the experiment using 8 kinds of metal ( $\mathrm{Al}, \mathrm{Au}, \mathrm{Cr}, \mathrm{Ni}, \mathrm{Pt}, \mathrm{Ti}, \mathrm{Zn}$, and $\mathrm{Zr}$ ) and polystyrene particle, the net charge generated was shown to be compared fairly well with the conventional simple condenser model based on metal-to-metal contact model in terms of contact potential difference in its order of magnitude. Although a clear correlation was not obtained between the measured charge density and the work functions of metal targets because of a large scatter in the data, a strong relationship between the charge density and contact area was found. The underlying mechanisms for the relationship is not known at this moment; however the finding gives a good hint for the next attempt.
\end{abstract}

Keywords: contact electrification, tribo-charging, tribo-electrification, adhesive force measurement, electrostatic adhesion, Coulomb's law

\section{Introduction}

The first establishment of so-called reversed square law of the electrostatic interaction is credited to Coulomb's direct measurement (1785) by using torsion balance ${ }^{1)}$ (although there might be some arguments in the context of science history, but it is

\footnotetext{
This paper, appeared originally in Japanese in J. Soc. Powder Technology Japan 43, 174-180(2006), is Published in KONA Powder and Particle Journal with the permission of the editorial committee of the Soc. Powder Technology, Japan.

1 1-236 Tangi-cho, Hachioji Tokyo, 192-8577 JAPAN

* Conesponding authe

TEL \& FAX: +81-(0) 42-691-8169

E-mail: tatsushi@t.soka.ac.jp
}

beyond the subject of this technical paper). 200 yeas after Coulomb, the first attempt of the direct measurement of the electrostatic interaction working on small single particles, like powders, was demonstrated by Arakawa et al. ${ }^{2}$ by using precise balance modified from adsorption balance system. Their study was conducted in the context of direct evaluation of powder adhesion, after which they applied such direct measurement of force between single particles to small particle size region, down to $100 \mu \mathrm{m}$. With regard to the electrostatic interaction, they successfully measured force curves satisfying the reversed square law. Note here that the Coulomb's law had been one of the most established and essential laws in physics; therefore, they did not 're-discover' it. The 
When the adhesive force of dry powder is discussed, any textbook on powder technology describes that there are three kinds of forces as liquid bridge force, intermolecular force and electrostatic force, in which the order of magnitude of the forces decreases in the order. It is generally understood that electrostatic force can be negligible. On the other hand, however, there are a lot of technologies to control powder by electrostatic force. It is found not exclusive only on the technology of electrophotograpy. There are more than few discussions attributing unexpected kinetic behaviors of powder to electrostatic force in industrial processes (or in labscaled experiments) of dry powder handling. Again these situations are incompatible to the discussions that the electrostatic force is negligible.

One of the major reasons of this kind of controversial situation where whether the electrostatic interaction is negligible or not in actual dry powder processes is due to the difficulty of evaluation or prediction of the amount of charge on powder. If the amount of charge on particles is estimated, the force acting on the particle can be determined by the Coulomb's law. The reverese is true that if the adhesive force acting on a particle can be measured precisely, the amount of charge on the particle can be reversely calculated and determined. Additionally the measurement can give new information on electrostatic charge generation on a single particle if the contact and separation of the particle against a target are well controlled.

As mentioned above, there are three mechanisms for the adhesive force working on a particle. On the other hand, however, the capillary force works only after the liquid bridge is generated when the contact of a particle is accomplished, i.e., this interaction has hysteresis before and after the contact. The intermolecular force is very sensitive to the surface distance; therefore, it decays rapidly with an increase of contact distance comparing to the electrostatic interaction. With considering these characteristics of three kinds of interactions, the information of the electrostatic interaction can be separated from a measurement of a force curve working on a single particle. Note here that it is important to record 'force curve,' i.e., it is necessary to evaluate not only the maximum adhesive force but also the force with the surface distance simultaneously and precisely.

A candidate of an instrument realizing such measurement may be atomic force microscope (AFM), which is now widely used in various applications. Though it is a kind of a ultra precise stylus method in which a microscopic bending of a small cantilever is measured with a principle of light lever. It was originally a microscope to observe surface morphology, the use of its 'force curve mode' makes it possible to measure the force working between a particle, which is fixed on the tip of a cantilever, and a flat target with precise control or measurement of the surface distance. It may be also noted that such measurements can be performed in both liquid phase and gas phase with the system.

In this work, 'force curve' of a single particle in dry system was measured using AFM. Based on the results of the measurements, evaluations on electrostatic interaction and charge generation on a single particle due to contacts to metal flat target will be discussed in details.

\section{Experimental Method}

The AFM used is JSPM-4210, JEOL. On the tip of a cantilever, a single spherical particle was fixed by epoxy adhesion as normal way. Sample particles were glass beads of around $30 \mu \mathrm{m}$ in diameter, polystyrene spheres of around 30 and $100 \mu \mathrm{m}$ in diameter. Before use, they were cleaned with ethanol and dried. As targets, a sample stage of the AFM, coated by gold on the surface, was used as a flat gold target. Additionally, 8 different metal plates $(\mathrm{Al}, \mathrm{Au}, \mathrm{Cr}, \mathrm{Ni}, \mathrm{Pt}, \mathrm{Ti}$, $\mathrm{Zn}, \mathrm{Zr}$ ), mirror finished by buffing, were used. Table 1 summarizes work function values cited ${ }^{3)}$ for these metals and the results of the measurement of their surface roughness (Surfcom, Tokyo Seimitsu). The force curve measurements were performed in an ambient air. The humidity for the measurement was not controlled but monitored, which was in the range of 25 to $45 \%$ R.H.

Table 1 Surface roughness and work functions ${ }^{3)}$ of 8 kinds of metal plate samples

$\begin{array}{lllllllll}\text { metal } & \mathrm{Al} & \mathrm{Au} & \mathrm{Cr} & \mathrm{Ni} & \mathrm{Pt} & \mathrm{Ti} & \mathrm{Zn} & \mathrm{Zr}\end{array}$

$\begin{array}{lllllllllll}\text { Roughness }(\mathrm{Ra})[\mu \mathrm{m}] & 0.14 & 0.02 & 0.12 & 0.01 & 0.09 & 0.11 & 0.02 & 0.04\end{array}$

Work function[eV] $\quad 4.17 \quad 5.38 \quad 4.5 \quad 5.2 \quad 5.64 \quad 4.33 \quad 3.63 \quad 4.05$

\section{Results and Discussion}

Fig. 1 shows an example of the measured force curve with a $35 \mu \mathrm{m}$ glass bead. This is the result of the force curve reached to a stable condition after repeated contacts of tens of times. Although the actual 


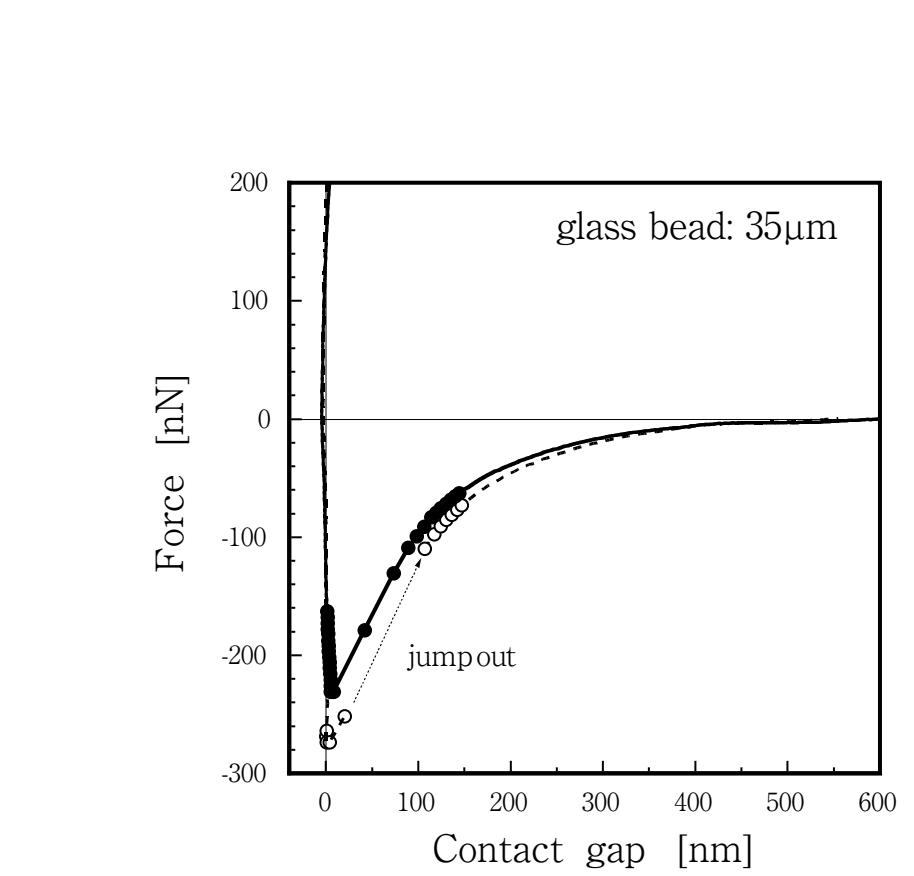

Fig. 1 An example of the obtained force curve against AFM stage as a target made of gold (glass bead, $35 \mu \mathrm{m}$ ).

data were recorded discretely or digitally, the number was more than 2000 for each way of approach or separation, thus the curves are drawn with only lines:
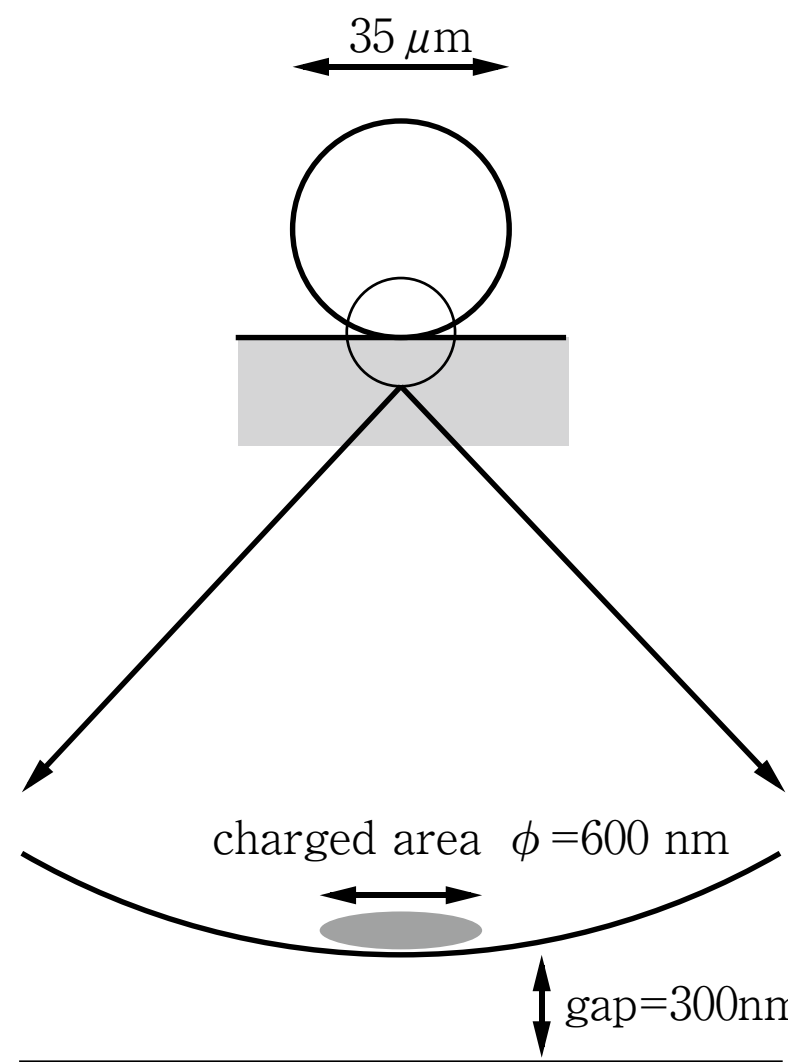

Fig. 2-a

Fig. 2 Schematic illustration of (a) contact area, and of (b) a comparison of point-to-point charge interaction and disk-to-disk interaction. only several important points just around jump-in and jump-out are plotted with markers. The curves in approach and separation are the same; therefore, no liquid bridge force is obtained in the measurement. The estimated intermolecular force for the separation distance range was negligible to the order of the measured force; therefore, it is concluded that the obtained force curve shows only the electrostatic interaction due to electrostatic charge generated on the tip of the particle by contact with the target.

Discussed in the next step is how the force curve can be evaluated. If a sphere is charged on its surface uniformly, the electric field around the particle is equivalent to that generated by a point charge of the total amount located on the center of the sphere. In this case the image force working between the center charge and its 'image' located inside of the metal target can be calculated as the electrostatic interaction working on the particle. Still, it should be noted that the polarization effect is neglected in the calculation $^{4)}$.

Such a calculation with a center charge is only for a uniformly charged sphere. In the case of the force curve to be evaluated here, the charge after repeated contacts between the particle and the target is localized only on the contact area. Therefore the use of reversed square law for the center charge and its image is not reasonable. On the other hand, an approximation with a point charge localized on the tip of a particle gives force divergent with gap zero, which is also unreasonable.

If the contact area on the tip of a particle is sufficiently small, comparing to the curvature of the particle, the contact area can be approximated with a flat disk. With an assumption of uniform charge on the flat disk, the interaction between the disk charge and its image is calculated. A schematic illustration of this approximation is shown in Fig. 2.

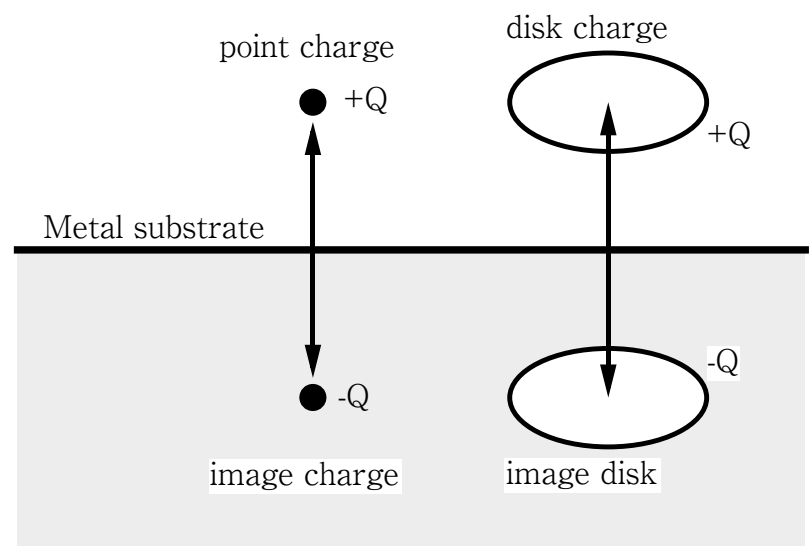

Fig. 2-b 
arteaforce working between the disks of radius $a$ and surface charge densities $\sigma$ and $-\sigma$, which are weparated coaxially with a distance $h$, is calculated as follows.

$$
\begin{aligned}
& F=\frac{\sigma^{2}}{4 \varepsilon_{0}} \int_{0}^{a} \int_{0}^{a} \int_{0}^{2 \pi} \int_{0}^{2 \pi} \frac{h r_{1} r_{2}}{R^{3}} d r_{1} d r_{2} d \varphi d \theta \\
& R=\sqrt{h^{2}+r_{1}^{2}+r_{2}^{2}-2 r_{1} r_{2} \cos (\theta-\varphi)}
\end{aligned}
$$

The force is normalized by eq.(3) with the total charge on the disk $\mathrm{q}=\sigma \pi a^{2}$ as,

$F_{N}=F /\left(\frac{q^{2}}{4 \pi \varepsilon_{0} a^{2}}\right)$

Then the normalized force is given as,

$$
F_{N}=\frac{1}{\pi^{2} a^{2}} \int_{0}^{a} \int_{0}^{a} \int_{0}^{2 \pi} \int_{0}^{2 \pi} \frac{h r_{1} r_{2}}{R^{3}} d r_{1} d r_{2} d \varphi d \theta
$$

This cannot be integrated analytically. Thus, after evaluating this numerically, an analytical approximation applicable for parameter-fitting afterward is found by a trial-and-error.

$F_{N}=\frac{2}{2(h / a)^{2}+(h / a)+1}$

Fig. 3 shows the comparison between the result of numerical integration of eq.(4) and the approximation of eq. (5). A good approximation is shown for the entire range, although an error goes up to $20 \%$ only with a very short gap. For comparison, the result of the approximation with a point charge, located on the tip of the particle, is also shown. Because the force increases rapidly and goes to infinity for the short gap naturally, this calculation is useless to evaluate the obtained force curve.

Let $d$ be the distance between a particle and a flat metal target. Because the distance between the disk

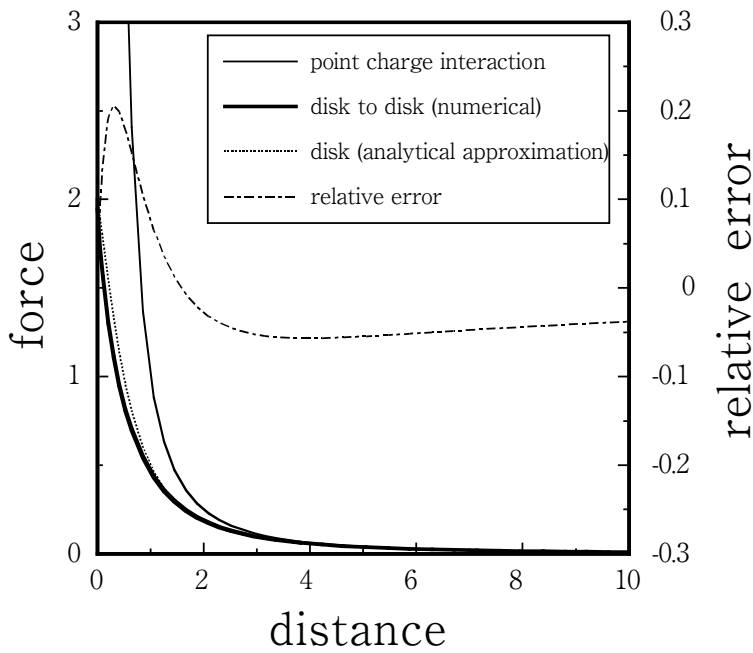

Fig. 3 Numerically calculated disk-to-disk charge interaction, compared with point-to-point interaction as well as the analytical approximation. Also the relative error of the approximation is shown. charge and its image is $h=2 d$, from eq.(5) the force working on the disk charge is given as,

$F=\frac{q^{2}}{4 \pi \varepsilon_{0} a^{2}} \cdot \frac{2}{8(d / a)^{2}+2(d / a)+1}$

Note that the force goes to $F=\pi \sigma^{2} \sigma^{2} / 2 \varepsilon_{0}$ with the limit of $d \rightarrow 0$. This corresponds to the force between sufficiently large disks, comparing to the gap.

As discussed above, the point charge approximation gives the interaction divergent with the short gap. To avoid this difficulty, sometimes another approximation is used with a point charge located inside of the sphere with a small distance $a$, implied to the size of the contact area, from the tip of the sphere. For this case, however, the equivalent surface charge distribution on the particle corresponding to the point charge cannot be given simply. With this approximation, the force is evaluated with the following equation, which can be compared with eq.(6).

$F=\frac{q^{2}}{4 \pi \varepsilon_{0} a^{2}} \cdot \frac{1}{4(d / a)^{2}+8(d / a)+4}$

Fig. 4 shows an example of the evaluation of an obtained force curve, shown in Fig. 1. With using eq. (6), the regression analysis was applied to the data with parameters as the radius of charge area (disk) and the total amount of charge (on the disk). For comparison, a result of data fitting with the approximation of the point charge located on the tip of the particle is also shown. The point charge approximation obviously gives the force going to the infinity. On contrary, the disk charge approximation fits to the data for the entire range. From the results, it was concluded that the force curve obtained corresponds to the electrostatic interaction and the charge area on the particle can be expressed as a disk.

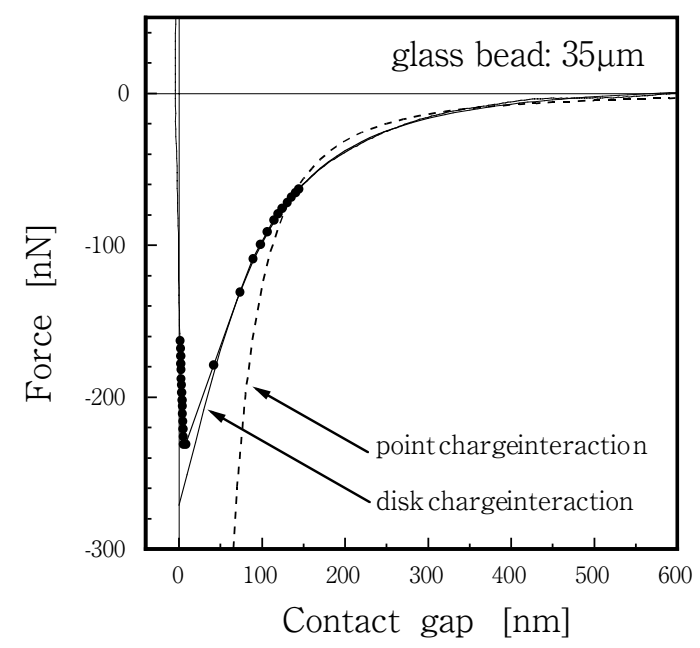

Fig. 4 Data from Fig. 1 with fitted line of disk-to-disk charge interaction. The case of point charge interaction is also shown, which doses not explain the data. 
paricie

Fign shows an example of the obtained data with poly styrene particle of $27.5 \mu \mathrm{m}$ in diameter. The result is wimilar to the case of the $35 \mu \mathrm{m}$ glass bead.

Next, Fig. 6 shows a result of polystyrene particle of $100 \mu \mathrm{m}$ in diameter. The result was completely different from other results of smaller particles shown above, i.e., the force curves in approach and separation were different to each other. Note that the results were obtained after repeated contacts, as was the case of the smaller particles, in which the force curves were measured stably. The big force in the separation was lost in the next approach, but appeared again in the next separation. This bigger force in the separation appeared after 'jump-out,' this is

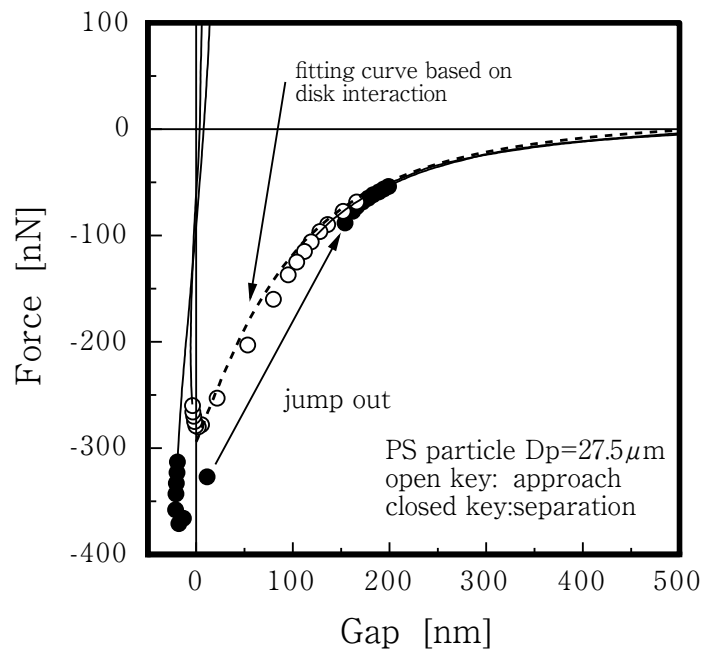

Fig. 5 Force curve of PS particle of $27.5 \mu \mathrm{m}$ against AFM stage with curve fitted.

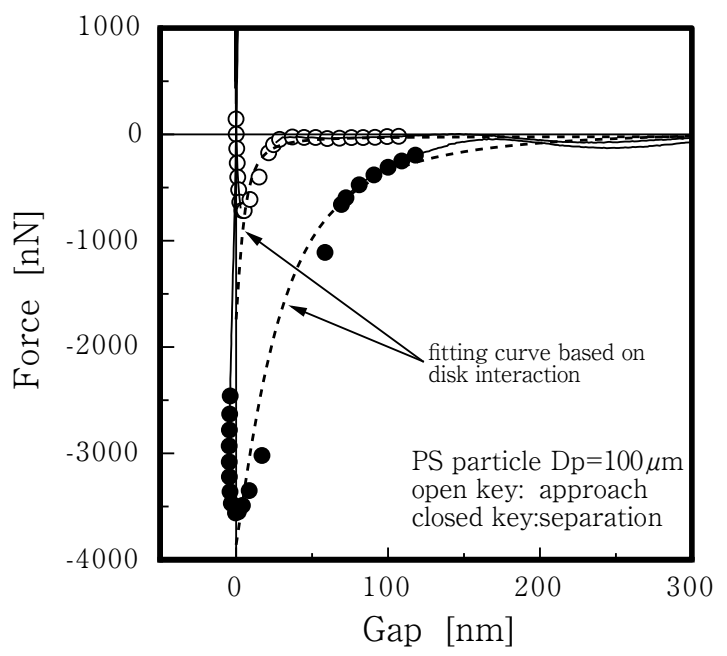

Fig. 6 Force curve of glass bead of $100 \mu \mathrm{m}$ against AFM stage. The curves for approach and separation are completely different to each other. The force in separation is significantly bigger than that in approach, although the data are taken after repeated contact, and it is repeatable. not due to the capillary force.

If these forces were of electrostatic interaction due to the charge on a particle, this result means that there is a process as follows: (1) in approach, the particle has a certain amount of charge, (2) in contact, the contact charge is generated on the particle, (3) as its result, the bigger force curve is obtained in the separation process, (4) however, in the separation process, somehow and somewhat the particle loses its charge, (5) and this process is repeatable.

On the other hand, however, this process does not correspond directly to our model of "charge relaxation due to gas discharge ${ }^{5,6)}$ because of the model based on Paschen's law of gas discharge where the specific gap of it is in $\mu \mathrm{m}$ range. This is far bigger than the range in the present measurement of the force curve. This point should be studied in more detail in the future.

Fig. 7 shows the results of the experiments with 8 kinds of metal target and polystyrene particle of 100 $\mu \mathrm{m}$ in diameter. The relationship between the work functions of the metal targets and the charge density is shown, where the generated charge on the particle was estimated by the data regression as discussed above from the force curve measurement. In the evaluation, the data in the separation process were used because they were considered to correspond to the amount of charge which is just generated by the contact event, as discussed above.

First of all, the data scattered considerably. We repeated experiments to improve this situation; however the reason is not known at this moment. Therefore, the correlations of charge density to metal work function are not considered here. However, it should

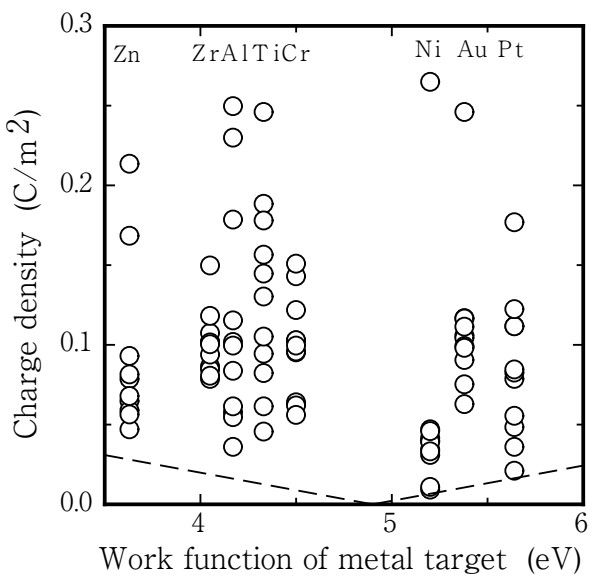

Fig. 7 Relationship of obtained charge density and work function of metal targets for $100 \mu \mathrm{m}$ PS particle. Dashed line shows the charge density expected from the condenser model with an assumption of $4.9 \mathrm{eV}$ for the effective work function of PS. 
bonnoted that the evaluated charge densities were extraordinary high as $10^{-2}$ to $10^{-1} \mathrm{C} / \mathrm{m}^{2}$ in its order of w magnitude. In our previous work of impact charging experiments ${ }^{7)}$, the obtained charge density with the same polystyrene particle of $100 \mu \mathrm{m}$ in diameter was in the order of $10^{-4} \mathrm{C} / \mathrm{m}^{2}$. In comparison the former is extremely high. From this discussion, it is suggested that the net charge transfer due to contact is obtained in the force curve measurement with AFM, whereas the charge after the charge relaxation due to gas discharge is obtained in the impact charging experiment. However, the order obtained in the present work is even higher than that estimated by the standard or conventional condenser charging model (or metal-to-metal contact model, or high-density-limit model ${ }^{8,9)}$ ), which is drawn for comparison in Fig. 7 with an assumption of $4.9 \mathrm{eV}$ as the effective work function of polystyrene. At this moment we can offer no theory to explain this. Therefore, possibilities of experimental errors or failures in the procedure of the force curve evaluation are still necessary to be studied.

Furthermore, we continued to repeat the measurements and accumulated more data. Fig. 8 shows the relationship between the evaluated surface charge density and the contact area. This figure shows only the result of $Z n$ target case. In Fig. 7, the charge density looked only as scattered, but in Fig. 8, it was found that the charge density follows a certain function or a correlation to the contact area. Fig. 9 shows the similar result for polystyrene particle of $30 \mu \mathrm{m}$ in diameter. In the data of Fig.9, the force curves, obtained with $30 \mu \mathrm{m}$ polystyrene particle and $\mathrm{Zn}$ target, were similar to the result shown in Fig.6, which showed a bigger interaction in separation than that in approach. Also they were different from the result

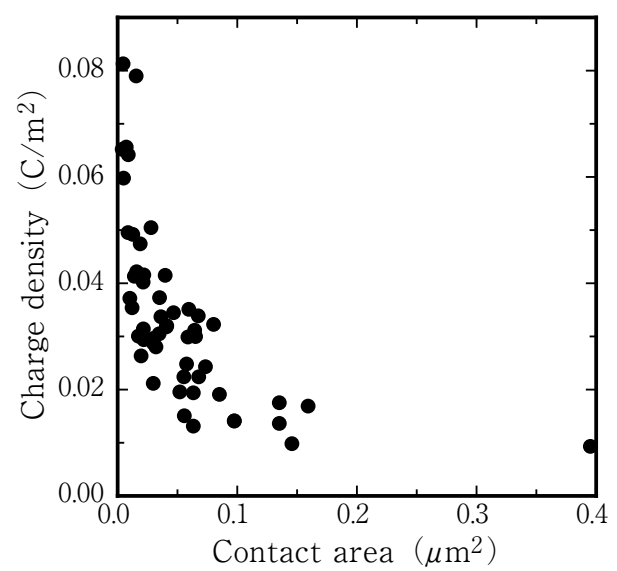

Fig. 8 The relationship between charge density and contact area (PS $100 \mu$ m particle, $\mathrm{Zn}$ target.)

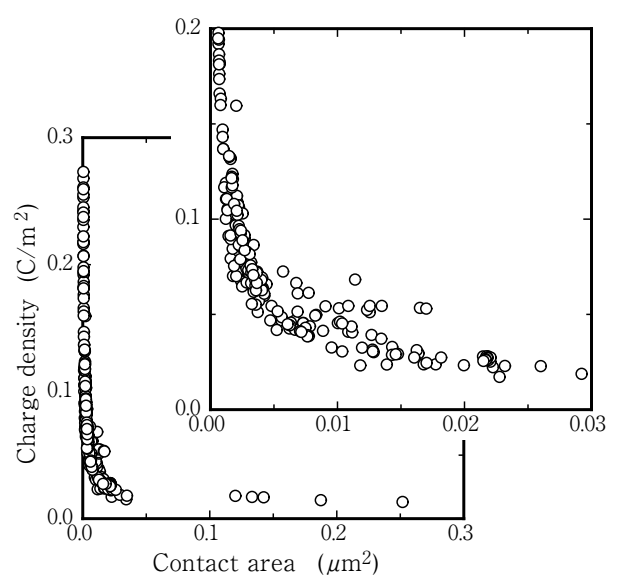

Fig. 9 The relationship between charge density and contact area (PS $30 \mu \mathrm{m}$ particle, $\mathrm{Zn}$ target.)

shown in Fig. 5, even though the particle size was similar to the case of Fig.5. Again it is not known at present that whether these kinds of different 'modes' in the force curve are resulted only from the difference in targets. This is an interesting point to be explored in the future in more detail.

With regard to Figs. 8 and $\mathbf{9}$, it was also interesting that the relationship or correlation between the charge density and the contact area was found although the charge density data seemed just as scattered as Fig. 7. In the future study, the detailed studies are necessary to explore whether the relationship has any significant physical meaning or there still are any errors or artifacts in the measurements or evaluation procedures. It would be interesting if this kind of investigation could be a new key to open the new door to understand the contact charging of a single particle.

\section{Conclusions}

The electrostatic interaction due to charge generated by repeated contacts between a single spherical particle glued on the tip of the cantilever of AFM and flat metal targets were measured with the force curve measuring mode of AFM. It was shown that the electrostatic force working on the particle can be evaluated independently and separately from the capillary force and the intermolecular force by evaluating the obtained force curve, not only the maximum contact force. To evaluate the force curve by electrostatic interaction, a disk charge approximation, in which the contact area on the tip of a particle is evaluated as a small and flat disk, was studied. The force between a disk and its image was numerically calculated, and a good analytical approximation was 
foungto express the force as a function of the contact gap. B. comparing the data and the obtained theorefical force curve, it was shown that the measured force curve corresponds the electrostatic interaction and that the surface charge density and the size of the contact area (radius of the disk) can be estimated by the data fitting. The obtained or evaluated surface charge density was in the order of $10^{-2} \mathrm{C} / \mathrm{m}^{2}$, which was significantly bigger than $10^{-4} \mathrm{C} / \mathrm{m}^{2}$ obtained in our previous work with impact charging experiments with the same particles. This fact suggested that the charge obtained in the AFM-force curve measurement is corresponding to the net charge which is just generated by the contact before the charge relaxation due to gas discharge takes place. With the detailed experiments using 8 kinds of metal target, any clear correlation between the generated charge (density) and the metal work function could not be found. The obtained charge density was bigger than that predicted by the standard condenser charging model. The reason why such kinds of results were obtained is not known at this moment. The obtained charge density scatted significantly with many measurements. Afterwards, however, it was found that the data did not simply scattered but there was a certain relationship between the charge density and the contact area. However, the significance of this relationship is unclear at this point. We hope that the clarification of this point would become the clue to the detailed study on the contact charging of a particle in the future.

\section{Acknowledgements:}

This work was supported by IFPRI (International Fine Particle Research Institute). The authors appreciate Mr.T.Sueyoshi, JEOL, for his helpful suggestions on the force curve measurement by AFM. The authors also appreciate Nihon Micro Coating for their help in finishing the surface of the flat metal plates as the target.

\section{Nomenclature}

$a$ : radius of charged disk

[m]

$d$ : distance between particle and target

[m]

$F$ : force

[N]

$F_{N}$ : normalized force

[-]

$h$ : distance

[m]

$q$ : charge

[C]

$R$ : distance

[m]

$r$ radius

[m]

$\varepsilon_{0}$ : dielectric constant of space

$[\mathrm{F} / \mathrm{m}]$

$\phi$ : angle

[rad]

$\sigma:$ surface charge density

$\left[\mathrm{C} / \mathrm{m}^{2}\right]$

$\theta$ : angle

[rad]

\section{References}

1) e.g., Roller, D., DHD. Roller: "The development of the concept of elctric charge”, In: J. B.Contant ed. Harvard Case Histories in Experimental Science vol.2, Cambrige, USA: Harvard University Press, pp.541-633 (1970).

2) Arakawa, M., S. Yasuda S: "The measurement of interaction force on microparticles”, Zairyo, 26, pp.858-562 (1977).

3) Inst. Electros. Jap. ed.: "Seidenki Handbook”, Ohmusha, Tokyo (1981).

4) e.g., Matsuyam, T., H. Yamamoto: "The electrostatic force between a charged dielectric particle and a conducting plane”, J. Soc. Pow. Tech., Jap., 34, pp.154-159 (1997).

5) Matsuyama, T., H. Yamamoto: "Charge relaxation process dominates contact charging of a particle in atmospheric conditions”, J. Phys. D: Appl. Phys., 28, pp.2418-2423 (1995).

6) Matsuyama, T., H. Yamamoto: "Charge relaxation process dominates contact charging of a particle in atmospheric conditions II. general model”, J. Phys. D: Appl. Phys., 30, pp.2170-2175 (1996).

7) Matsuyama, T., M. Ogu, H. Yamamoto, JCM. Marijnissen, B. Scarlett: "Impact charging experiments with single particles of hundred micrometre size”, Pow. Tech., 135/136, pp.14-22 (2003).

8) Schein, LB., M. LaHa, G. Marshall: "Electrostatic charging of two insulating powders”, J. Appl. Phys., 69, pp.6817-6826 (1991).

9) Schein, LB.: "Recent advances in our understanding of toner charging”, J. Electros., 46, pp.29-36 (1999). 


\section{Author's short biography}

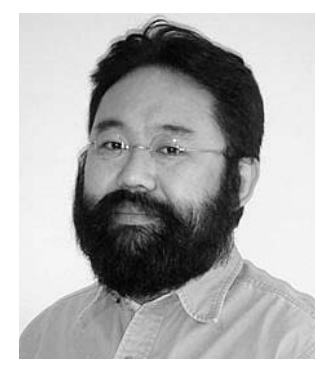

\section{Tatsushi Matsuyama}

Dr.Tatsushi MATSUYAMA is Professor of Department of Environmental Engineering for Symbiosis, Faculty of Engineering, Soka University. He graduated from Department of Chemical Engineering, the University of Tokyo, in 1987. He received the $\mathrm{PhD}$ degree in chemical engineering from the University of Tokyo in 1995, with the thesis on the mechanism of impact charging of single particles. Since then, he has been contributing in the field of powder technology, and his major research interests have been still in the areas of powder and electrostatics. He spent his sabbatical leaves as visiting researcher at the Delft University of Technology, the Netherlands, with Prof.B.Scarelett's group in 1998, and at the Institute of Particle Science and Engineering, Leeds University, UK, with Prof.M.Ghadiri's group in 2007. He is currently the editor-in-chief of Journal of the Institute of Electrostatics Japan.

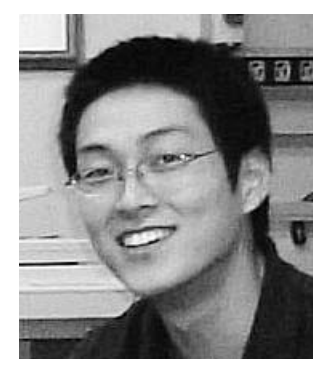

\section{Masaaki Ohtsuka}

Masa-aki OHTSUKA obtained his BSc (2005) and MSc (2007) from Faculty of Engineering, Soka University and its Graduate School of Engineering.

\section{Hideo Yamamoto}

Dr.Hideo YAMAMOTO is Professor of Department of Environmental Engineering for Symbiosis, Faculty of Engineering, Soka University. He graduated from the Department of Chemical Engineering, the University of Tokyo, in 1967. He received the Dr.Eng. degree from the University of Tokyo in 1979. He joined Department of Chemical Engineering, the University of Tokyo in 1976, and in 1989 to become an Ass.Prof. at the Institute of Industrial Science, the University of Tokyo. He was a visiting researcher at the Delft University of Tecunology, in the Netherlands from 1984 to 1986. He joined the Department of Bioengineering at Soka University, as a Professor in 1991. His field and interesting is in particle technology and applied electrostatics. His current researches are particle separation from gases, particle size analysis, production and application of nano-particles, chemical reaction in surface corona plasma and its application for pollution control. 